Obere Extremität 2018 $\cdot 13: 196$

https://doi.org/10.1007/s11678-018-0473-1

Online publiziert: 6. August 2018

(c) Springer Medizin Verlag $\mathrm{GmbH}$, ein Teil von Springer Nature 2018

CrossMark
M. Beirer ${ }^{1}$ L. Willinger ${ }^{2} \cdot$ L. Lacheta $^{2} \cdot$ A. Buchholz $\cdot$ A. Lenich ${ }^{3} \cdot$ A. B. Imhoff ${ }^{2}$. S. Siebenlist ${ }^{2}$

' Klinik und Poliklinik für Unfallchirurgie, Klinikum rechts der Isar, Technische Universität München, München, Deutschland

${ }^{2}$ Abteilung für Sportorthopädie, Klinikum rechts der Isar, Technische Universität München, München, Deutschland

${ }^{3}$ Klinik für Orthopädie, Unfall-, Handchirurgie und Sportorthopädie, Helios Klinikum München West, München, Deutschland

\section{Erratum zu: Subjektives und funktionelles Outcome nach akut-ligamentärer Ellenbogenluxation}

\section{Operative vs. konservative Therapie}

\section{Erratum zu:}

Obere Extremität 2018

https://doi.org/10.1007/s11678-018-

0468-y

In dem ursprünglichen Artikel wurde der Name des Autors L. Lacheta falsch geschrieben. Bitte beachten Sie die korrigierte Schreibweise.

\section{Korrespondenzadresse}



PD Dr. med. S. Siebenlist Abteilung für Sportorthopädie, Klinikum rechts der Isar, Technische Universität München

Ismaningerstraße 22, 81675 München, Deutschland sebastian.siebenlist@ mri.tum.de 